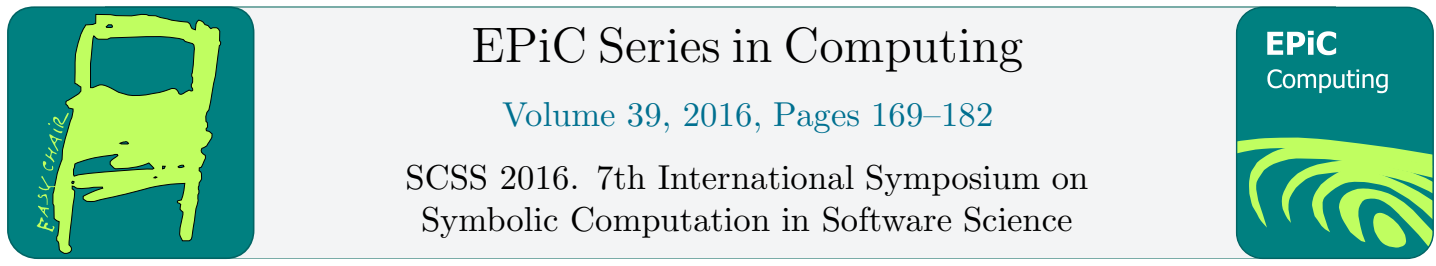

\title{
Bit-size reduction of triangular sets in two and three variables
}

\author{
Yamashita Tetsuro $^{1}$ and Dahan Xavier ${ }^{2 * \dagger}$ \\ ${ }^{1}$ Fujitsu company \\ ${ }^{2}$ Faculty of General Educational Research, Ochanomizu University, Tokyo, JAPAN. \\ xdahan@gmail.com
}

\begin{abstract}
At ISSAC 2004 [4] was introduced a transformation of a triangular lexicographic Gröbner basis generating a radical ideal of dimension zero, to a triangular family of polynomials generating the same ideal, which is no more a Gröbner basis but has significantly smaller coefficients in term of bit-size. We attempt in this article to extend this transformation to triangular sets that do not generate a radical ideal. We manage to treat the case of $n=2$ variables, and in some extent the case of $n=3$ variables. It resorts to an extra operation, the squarefree factorization; nevertheless this operation based on gcd benefits of efficient algorithms. When the number of variables $n$ is greater than 2, more serious difficulties occur and are discussed for $n=3$. An implementation in Maple in the case $n=2$ confirms the expected reduction of the bit-size coefficients.
\end{abstract}

\section{Introduction}

A reduced lexicographic Gröbner basis for the monomial order $x_{1} \prec x_{2} \prec \cdots \prec x_{n}$ over a field $K$ is triangular if all of its polynomials have a leading monomial that is a pure power of a variable, which are all pairwise distinct. It implies that the basis is a regular sequence. When there are $n$ polynomials $T_{1}, T_{2}, \ldots, T_{n}$ in such a Gröbner basis, and they are assumed to be ordered so that $\operatorname{LM}\left(T_{1}\right) \prec \operatorname{LM}\left(T_{2}\right) \prec \cdots$ it can be written as:

$$
\mathbf{T}\left\{\begin{array}{l}
T_{n}\left(x_{1}, x_{2}, \ldots, x_{n-1}, x_{n}\right)=x_{n}^{d_{n}}+a_{n, n-1}\left(x_{1}, \ldots, x_{n}-1\right) x_{n}^{d_{n-1}} \ldots \\
T_{n-1}\left(x_{1}, \ldots, x_{n-1}\right)=x_{n-1}^{d_{n-1}}+\cdots \\
\vdots \\
T_{1}\left(x_{1}\right)=x_{1}^{d_{1}}+\cdots
\end{array}\right.
$$

where $\cdots$ stands for lower terms for $\prec$. It is common to call such a family triangular sets. Such simple data structures are at the core of the triangular decomposition method (see [8,

\footnotetext{
* Commissioned by "Strategic Information and Communications R\&D Promotion Programme (SCOPE), No. 0159-0016" Ministry of Internal Affairs and Communications, JAPAN.

${ }^{\dagger}$ And supported by Kakenhi research grant No.15K16003
} 
$1,2,7]$ among many others) to solve and manipulate polynomial systems. Note that within the assumptions made, i.e. a finite number of solutions, monic polynomials as those in (1) naturally appear, or, if not, a mere inversion in the base field $K$ allows to reduce to monic polynomials. We focus mainly on the case of $K=\mathbb{Q}$ or $K=\mathbb{F}_{p}$ (see "Motivation" below). The transformation introduced in [4]

$$
T_{1} \rightarrow N_{1}, \quad T_{i} \rightarrow N_{i}:=\left(\prod_{\ell=1}^{i-1} \frac{\partial T_{\ell}}{\partial x_{\ell}}\right) T_{i} \bmod \left(T_{1}, \ldots, T_{i-1}\right),
$$

allows to compute at a cheap cost polynomials with smaller coefficients. More precisely, the former has coefficients bit-size that grows at most in $O\left(d^{2 n}\right)$ where $d=\prod_{i} \operatorname{deg}_{x_{i}}\left(T_{i}\right)$, whereas the latter has coefficients bit-size that grows at most in $O\left(d^{n}\right)$. The two ideals $\left\langle T_{1}, \ldots, T_{n}\right\rangle$ and $\left\langle N_{1}, \ldots, N_{n}\right\rangle$ are equal but the second family is no longer a Gröbner basis. Such a transformation is implemented in Maple in the RegularChains library under the name DahanSchostTransform.

Motivation Despite not Gröbner bases, those families of polynomials find applications in modular methods, where instead of lifting the coefficients of $T_{i}$, we lift the coefficients of $N_{i}$ which saves a significant amount of time. The figure below displays the concept of a modular method for a triangular decomposition. Instead of lifting directly the triangular sets modulo $p$ denoted $\mathbf{t}^{(i)}=\left(t_{1}^{(i)}\left(x_{1}\right), t_{2}^{(i)}\left(x_{1}, x_{2}\right), \ldots, t_{n}^{(i)}\left(x_{1}, \ldots, x_{n}\right)\right)$ in the figure below, it is more efficient to transform them into the polynomial families $\mathbf{n}^{(i)}=\left(\mathrm{n}_{1}^{(i)}\left(x_{1}\right), \ldots, \mathrm{n}_{n}^{(i)}\left(x_{1}, \ldots, x_{n}\right)\right)$ modulo $p$ by the operation (2), and then to lift them to polynomial systems $\mathbf{N}^{(i)}$ over $\mathbb{Q}$. This strategy is put into practice in [3] with precise complexity analysis, and implemented in the RegularChains library in Maple under the command name EquiprojectableDecomposition.

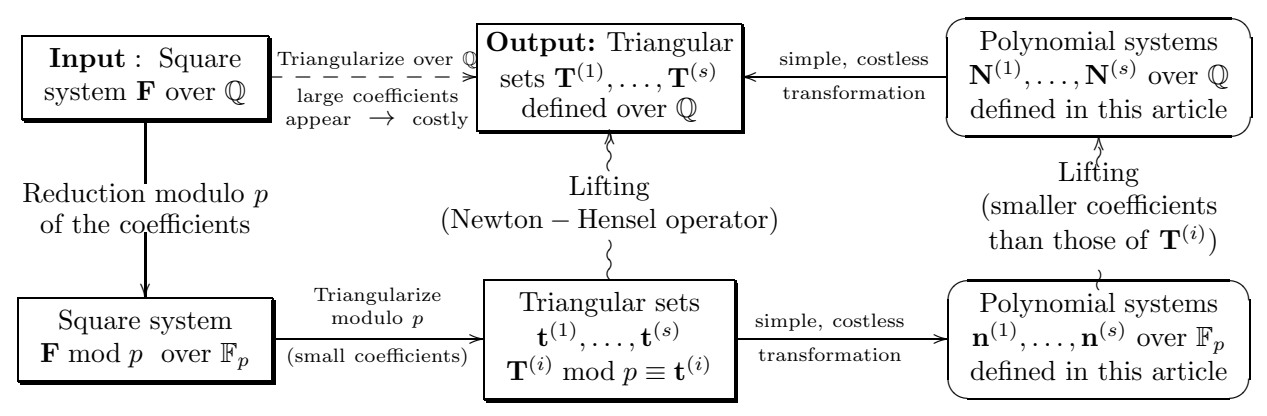

Figure 1: Prototype of a modular method modulo $p$ to triangularize a polynomial system $\mathbf{F}$ having a finite number of solutions to a family of $s$ triangular sets $\mathbf{T}^{(1)}, \ldots, \mathbf{T}^{(s)}$ (each of them is of the form $\left.\mathbf{T}^{(i)}=\left(T_{1}^{(i)}\left(x_{1}\right), T_{2}^{(i)}\left(x_{1}, x_{2}\right), \ldots, T_{n}^{(i)}\left(x_{1}, \ldots, x_{n}\right)\right)\right)$. Instead of lifting directly the coefficients of the triangular sets $\mathbf{t}^{(i)}$ as shown in the second column, transformation to the family of systems $\mathbf{n}^{(i)}$ is performed before lifting the smaller coefficients, as shown in the third column (see [3] for details).

As we will see below, some "interpolation" formula appearing in Theorems 1, 2 and Propositions 1, 3 require that the field $K$ is large enough: it is always enough to suppose that $|K|$ verifies, with notations introduced later, that $|K|>\operatorname{deg}_{x}\left(T_{1}\right) \operatorname{deg}_{y}\left(T_{2}\right)\left[\operatorname{deg}_{z}\left(T_{3}\right)\right]=d_{1} d_{2}\left[d_{3}\right]$.

When the polynomial system (1) does not generate a radical ideal, the formula (2) does not apply since $\prod_{\ell=1}^{i-1} \frac{\partial T_{\ell}}{\partial x_{\ell}}$ is no more necessarily invertible modulo $\left\langle T_{1}, \ldots, T_{i-1}\right\rangle$. In this paper, we extend those results to the simplest cases of $n=2$ and to some extent $n=3$ variables, as well as discussing the obstacles that prevent to consider a complete generalization to several variables. 
Previous work We emphasize here that our aim is a simple, algorithmically efficient formula to transform a system of type (1) to another triangular family of polynomials generating the same ideal, but with smaller coefficients. We are thus not interested by removing multiplicities, and the present work is not related to squarefree decomposition algorithms modulo a triangular set that aim to remove multiplicity like in [5].

Triangular decomposition algorithms indeed are not ideal-theoretic, but set-theoretic ones, therefore they do not necessarily represent the same ideal as the input system; the set of solutions is the same (or just almost the same in the case of regular chains). A first obstacle to represent non-radical ideals by triangular sets is that it is not always possible. But even when the ideal can be represented, in a broad term, by triangular sets, we are not aware of algorithms that allow to find these sets. As drawn in the conclusion, the reason of this difficulty can be explained by the lack of gcd algorithm over non-reduced rings of type $K[x] /\left\langle x^{2}\right\rangle$. The results presented can contribute to understand better what is going on in this situation.

\section{Case of two variables}

We consider two variables $x \prec_{\text {lex }} y$ and two polynomials $T_{1}(x) \in K[x]$ and $T_{2}(x, y) \in K[x, y] \backslash$ $K[x]$. The fact that $\left\{T_{1}, T_{2}\right\}$ is a reduced Gröbner basis of the zero-dimensional ideal that it generates implies the following elementary facts: $T_{1}$ is monic in $x$ and $T_{2}$ is monic in $y$. Let $d_{1}=\operatorname{deg}_{x}\left(T_{1}\right)$ and $d_{2}=\operatorname{deg}_{y}\left(T_{2}\right)$, and write $T_{2}(x, y)=y^{d_{2}}+c_{1}(x) y^{d_{2}-1}+\cdots+c_{d_{2}-1}(x) y+c_{d_{2}}(x)$. Each polynomial $c_{i} \in K[x]$ verifies $\operatorname{deg}_{x}\left(c_{i}\right)<d_{1}$.

\subsection{Review of the radical case}

In this paragraph, are briefly recalled the results of [4], valid for a radical ideal only, in the case of two variables. The radical assumption made there implies the following assumption:

Assumption 1. All the roots of $T_{1}$ are simple: $T_{1}(x)=\prod_{i=1}^{d_{1}}\left(x-\alpha_{i}\right)$, with $\alpha_{i} \neq \alpha_{j}$ if $i \neq j$.

Theorem 1. Under Assumption 1, define $p_{i}(y):=T_{2}\left(\alpha_{i}, y\right)$ and $M_{i}(x):=\prod_{\substack{0<j<d_{1} \\ j \neq i}}\left(x-\alpha_{j}\right)$.

1. $T_{2}$ can be written as a Lagrange interpolation polynomial:

$$
T_{2}(x, y)=\sum_{i=1}^{d_{1}} p_{i}(y) u_{i} M_{i}(x), \quad \text { where } \quad u_{i}=\sum_{j \neq i} \frac{1}{\alpha_{i}-\alpha_{j}} .
$$

2. Let $N_{2}(x, y) \equiv T_{1}^{\prime}(x) \cdot T_{2}(x, y) \bmod \left\langle T_{1}\right\rangle$ then

$$
N_{2}=\sum_{i=1}^{d_{1}} p_{i}(y) M_{i}(x)
$$

3. The polynomial $N_{2}$ verifies: $\left\langle T_{1}, N_{2}\right\rangle=\left\langle T_{1}, T_{2}\right\rangle$.

Remark 1. 1. It should be noted that $\left\{T_{1}, N_{2}\right\}$ is not a Gröbner basis.

2. The simplification yielding $N_{2}$ consists in suppressing the factors $u_{i}$ from the "Lagrange idempotent" $u_{i} M_{i}(x)$ in Equality (3). This is the reason why coefficients in $N_{2}$ are usually smaller than those of $T_{2}$. 
Example 1. Let $T_{1}(x)=(x-a)(x-b)(a \neq b \in K)$. Then $T_{2}(x, y)=T_{2}(a, y) \frac{x-a}{b-a}+T_{2}(b, y) \frac{x-b}{a-b}$ holds. As for $N_{2}$ :

$$
\begin{aligned}
& T_{1}^{\prime} T_{2}=\{(x-a)+(x-b)\}\left\{T_{2}(a, y) \frac{x-a}{b-a}+T_{2}(b, y) \frac{x-b}{a-b}\right\} \\
& T_{1}^{\prime} T_{2} \equiv T_{2}(a, y) \frac{(x-a)^{2}}{b-a}+T_{2}(b, y) \frac{(x-b)^{2}}{a-b} \equiv T_{2}(a, y)(x-a)+T_{2}(b, y)(x-b) \bmod \left\langle T_{1}\right\rangle \\
& \text { and according to Statement } 2 \text { of Theorem } 1 \quad N_{2}=T_{2}(a, y)(x-a)+T_{2}(b, y)(x-b) \text {. }
\end{aligned}
$$

\subsection{Preliminary toward a generalization to multiple roots}

We remove Assumption 1 and consider in this subsection a polynomial $T_{1}$ having multiple roots: $T_{1}(x)=\prod_{i=1}^{r}\left(x-\alpha_{i}\right)^{e_{i}}$ (where $\alpha_{i} \neq \alpha_{j}$ if $i \neq j$ and $e_{i} \in \mathbb{N}_{>0}$ ). The following proposition is a basic, straightforward application of the Chinese Remaindering Theorem.

Proposition 1. Let $p_{i}(x, y) \equiv T_{2}(x, y) \bmod \left\langle\left(x-\alpha_{i}\right)^{e_{i}}\right\rangle$ and $M_{i}(x):=\frac{T_{1}(x)}{\left(x-\alpha_{i}\right)^{e_{i}}} \in \bar{K}[x]$. Note that $\operatorname{deg}_{y}\left(p_{i}(y)\right)=\operatorname{deg}_{y}\left(T_{2}(x, y)\right)=d_{2}$. The following assertions are satisfied:

1. There exist $u_{i} \in \bar{K}[x]$ such that: $\quad \sum_{i=1}^{r} u_{i} M_{i}=1, \quad u_{i} \in \bar{K}[x]$.

2. $T_{2} \equiv \sum_{i=1}^{r} p_{i} u_{i} M_{i} \bmod \left\langle T_{1}\right\rangle$ (or equivalently $\left.T_{2}=\mathrm{NF}_{\left\langle T_{1}\right\rangle}\left(\sum_{i=1}^{r} p_{i} u_{i} M_{i}\right)\right)$.

Example 2. Given $T_{1}:=(x-1)^{2}(x-2)^{3}$, let $p_{1}=y+x, p_{2}=y+x^{2}$. To compute $T_{2}$ from the previous Proposition, we need $M_{1}=(x-2)^{3}, M_{2}=(x-1)^{2}$. The Extended Euclid Algorithm provides the equality $(2-3 x)(x-2)^{3}+\left(3 x^{2}-14 x+17\right)(x-1)^{2}=1$, so that with $u_{1}=2-3 x, u_{2}=3 x^{2}-14 x+17$, holds $u_{1} M_{1}+u_{2} M_{2}=1$.

$$
\begin{aligned}
T_{2} & \equiv p_{1} u_{1} M_{1}+p_{2} u_{2} M_{2} \bmod \left\langle T_{1}\right\rangle \\
& \equiv(2-3 x)(x-2)^{3}(y+x)+\left(3 x^{2}-14 x+17\right)(x-1)^{2}\left(y+x^{2}\right) \bmod \left\langle T_{1}\right\rangle \\
& \equiv y+3 x^{6}-23 x^{5}+68 x^{4}-96 x^{3}+65 x^{2}-16 x \bmod \left\langle T_{1}\right\rangle \\
& \equiv y+x^{4}-7 x^{3}+19 x^{2}-20 x+8 \bmod \left\langle T_{1}\right\rangle
\end{aligned}
$$

We can verify that $T_{2} \equiv p_{1} \bmod \left\langle(x-1)^{2}\right\rangle, T_{2} \equiv p_{2} \bmod \left\langle(x-2)^{3}\right\rangle$.

Proposition 2. Let $F(x)=\sum_{i=1}^{r} M_{i}(x) \in \bar{K}[x]$, we have:

$$
F(x) \cdot T_{2}(x, y) \equiv \sum_{i=1}^{r} p_{i}(y) M_{i}(x) \bmod \left\langle T_{1}(x)\right\rangle .
$$

Proof. By Proposition 1, $T_{2}(x, y) \equiv \sum_{i=1}^{r} p_{i}(y) u_{i}(x) M_{i}(x) \bmod \left\langle T_{1}(x)\right\rangle$ holds. Regarding that $\sum_{i=1}^{r} u_{i}(x) M_{i}(x)=1$, we have:

$$
F u_{i} M_{i}=\left(\sum_{i=1}^{r} M_{i}\right) u_{i} M_{i} \equiv u_{i} M_{i}^{2} \equiv\left(1-\sum_{j \neq i} M_{j}\right) M_{i} \equiv M_{i} \bmod \left\langle T_{1}\right\rangle .
$$

Therefore, $\quad F \cdot T_{2} \equiv F\left(\sum_{i=1}^{r} p_{i} u_{i} M_{i}\right) \equiv \sum_{i=1}^{r} p_{i}\left(F u_{i} M_{i}\right) \equiv \sum_{i=1}^{r} p_{i} M_{i} \bmod \left\langle T_{1}\right\rangle$.

Corollary 1. Defining $N_{2}(x, y):=\mathrm{NF}_{\left\langle T_{1}\right\rangle}\left(F(x) \cdot T_{2}(x, y)\right)$, holds: $\quad\left\langle T_{1}, T_{2}\right\rangle=\left\langle T_{1}, N_{2}\right\rangle$.

Proof. The inclusion $\left\langle T_{1}, T_{2}\right\rangle \supset\left\langle T_{1}, N_{2}\right\rangle$ is clear.

Since $T_{1}$ and $F$ are relatively prime, by the extended Euclid algorithm there exists $u, v \in$ $\bar{K}[x]$ such that $u T_{1}+v F=1$. Moreover by definition, there exists $A \in \bar{K}[x, y]$ such that $N_{2}=F T_{2}+A T_{1}$. As a result, $T_{2}=u T_{1} T_{2}+v F T_{2}=u T_{1} T_{2}+v\left(N_{2}-A T_{1}\right) \in\left\langle T_{1}, N_{2}\right\rangle$. Thus $\left\langle T_{1}, T_{2}\right\rangle \subset\left\langle T_{1}, N_{2}\right\rangle$. 
Remark 2. As in the case of Assumption 1, $\left\{T_{1}, N_{2}\right\}$ is not a Gröbner basis. The fact that $F \in K[x]$ is proved in Lemma 2, showing that $N_{2} \in K[x, y]$ thanks to Corollary 1.

In Example 2 the equation $N_{2} \equiv p_{1} M_{1}+p_{2} M_{2} \bmod \left\langle T_{1}\right\rangle$ yields $N_{2}=x^{3} y-5 x^{2} y+10 x y-$ $7 y+2 x^{4}-8 x^{3}+13 x^{2}-8 x$. In that toy example, it cannot be told that $N_{2}$ is simpler than $T_{2}$. But if the exponents $e_{i}$ of the factors of $T_{1}$ are sufficiently large it becomes clear that $N_{2}$ has smaller coefficients than those of $T_{2}$ as shown below.

Example 3. Set $T_{1}=(x-1)^{4}(x-2)^{5}$ and $p_{1}=y+x, p_{2}=y+x^{2}$. Let us compute $T_{2}$ and $N_{2}$.

$$
\begin{gathered}
T_{2}=y+20 x^{8}-254 x^{7}+1389 x^{6}-4265 x^{5}+8030 x^{4}-9480 x^{3}+6849 x^{2}-2768 x+480 \\
N_{2}=x^{5} y-9 x^{4} y+36 x^{3} y-74 x^{2} y+76 x y-31 y+2 x^{6}-14 x^{5}+46 x^{4}-84 x^{3}+81 x^{2}-32 x
\end{gathered}
$$

Remark 3. As under Assumption 1, this is explained by the fact that the factor $u_{i}$ is removed from $T_{2}$ in the formula 2. of Proposition 1 to obtain $N_{2}$.

\subsection{Computation of $N_{2}$ in a special case}

According to the previous subsection it is easy to compute $N_{2}$ if we know the polynomials $M_{i}=\frac{T_{1}}{\left(x-\alpha_{i}\right)^{e}}$. It suffices to compute the polynomial $F$ of Proposition 2 and $\mathrm{NF}_{\left\langle T_{1}\right\rangle}\left(F \cdot T_{2}\right)$ to obtain $N_{2}$. Unfortunately, to get the polynomials $M_{i}$ s, we are not aware of a simpler method than factorizing $T_{1}$, process which can be prohibitive especially over $\mathbb{Q}$. What we have in mind is a simple formula as in 2. of Theorem 1 to compute $N_{2}$ from $T_{1}, T_{2}$. To this end, we first consider a simpler assumption:

Assumption 2. The roots $\alpha_{i}$ below are pairwise distinct and have same multiplicity $e$ :

$$
T_{1}(x)=\prod_{i=1}^{r}\left(x-\alpha_{i}\right)^{e} \in K[x] .
$$

Lemma 1. Under Assumption 2, let $S(x):=\operatorname{sqf}\left(T_{1}(x)\right):=\prod_{i=1}^{r}\left(x-\alpha_{i}\right)$ be the squarefree part of $T_{1}$ and let $F_{e, j}(x):=\sum_{i=1}^{r}\left[M_{i}(x)\right]^{j / e}$. The following recurrence relation holds:

$$
F_{e, 1}(x)=S^{\prime}(x), \quad F_{e, \ell+1}=F_{e, 1} \cdot F_{e, \ell}-\frac{S}{\ell} \cdot F_{e, \ell}^{\prime} \quad(1 \leq \ell \leq e-1)
$$

In particular, all polynomials $F_{e, j} \in K[x]$.

Proof. By assumption $S=\prod_{i=1}^{r}\left(x-\alpha_{i}\right), \quad F_{e, \ell}=\sum_{i=1}^{r} \prod_{j \neq i}\left(x-\alpha_{j}\right)^{\ell}$. Moreover $F_{e, \ell}^{\prime}=$ $\ell \sum_{i=1}^{r} \sum_{k \neq i}\left(\prod_{j \neq i}\left(x-\alpha_{j}\right)^{\ell} / x-\alpha_{k}\right)$ holds, yielding:

$$
\begin{aligned}
F_{e, 1} \cdot F_{e, \ell} & =\sum_{i_{1}, i_{2}}\left(\prod_{j \neq i_{1}}\left(x-\alpha_{j}\right) \cdot \prod_{j \neq i_{2}}\left(x-\alpha_{j}\right)^{\ell}\right)=\sum_{i_{1}=i_{2}} \prod_{j \neq i_{1}}\left(x-\alpha_{j}\right)^{\ell+1} \\
& +\sum_{i_{1} \neq i_{2}}\left(x-\alpha_{1}\right)^{\ell+1} \cdots\left(x-\alpha_{i_{1}}\right)^{\ell} \cdots\left(x-\alpha_{i_{2}}\right) \cdots\left(x-\alpha_{r}\right)^{\ell+1}=F_{e, \ell+1}+\frac{S}{\ell} \cdot F_{e, \ell}^{\prime}
\end{aligned}
$$

which is what we wanted to prove.

Remark 4. This recurrence relation is used to compute $F=F_{e, e}$ of Proposition 2 from $S=$ $\operatorname{sqf}\left(T_{1}\right)$. The latter polynomial is obtained under Assumption 2 simply by taking the $e$-th root of $T_{1}$, and the former polynomial $F$ requires less than $O(A \log (A) \log (\log (A)))$ operations in $K$ to be computed, where $A=(e+1)\left(d_{1}-e\right)$ (see Appendix). 


\subsection{General case: need of squarefree factorization}

The same multiplicity Assumption 2 is here lifted, and we consider the general case $T_{1}=$ $\prod_{i=1}^{r}\left(x-\alpha_{i}\right)^{e_{i}}$ where the roots $\alpha_{i}$ are pairwise distinct. Let

$$
T_{1}=S_{1} S_{2}^{2} \cdots S_{n}^{n}
$$

be the squarefree decomposition of $T_{1}$. Recall that this factorization is straightforward to obtain by e.g. Yun's squarefree factorization [6, Algorithm 14.21, p. 385] valid in characteristic zero. Since in the case of $\operatorname{char}(K)=p>0$ we have assumed $|K|$ large enough, it is also valid in this case. The squarefree decomposition (6) can be obtained in $O\left(d_{1} \log \left(d_{1}\right)^{2} \log \left(\log \left(d_{1}\right)\right)\right)$ operations in $K$ by [6, Theorem 14.23], which is more efficient than computing a complete factorization of $T_{1}$ (and far more efficient if $\operatorname{char}(K)=0$ ).

Besides, it should be said that the squarefree decomposition has a good behavior under reduction modulo a prime $p$. If $p$ is large enough, the squarefree factors over $\mathbb{Q}$ correspond to the one obtained modulo $p$. This contrasts with the factorization into irreducibles where Chebotarev's density theorem $[6, \S 15.5$, page 429$]$ restrict to a given proportion the primes that yield a good behavior for the reduction modulo $p$.

Lemma 1 can be applied to each factor $S_{1}, S_{2}^{2}, \ldots, S_{n}^{n}$, for which Assumption 2 holds, in order to compute respective polynomials $F_{1,1}, F_{2,2}, \ldots, F_{n, n}$.

Lemma 2. The polynomial $F$ of Proposition 2 can be computed from the polynomials $S_{j} s$ and the polynomials $F_{j, j}$ and by the following formula

$$
F=\sum_{j=1}^{n} F_{j, j} T_{1} / S_{j}^{j} \quad \text { and } \quad F \in K[x] .
$$

Proof. Writing $S_{j}^{j}=\prod_{k_{j}}\left(x-\alpha_{k_{j}}\right)^{j}$,

$$
\sum_{j=1}^{n} F_{j, j} \frac{T_{1}}{S_{j}^{j}}=\sum_{j=1}^{n}\left(\sum_{k_{j}} \frac{S_{j}^{j}}{\left(x-\alpha_{k_{j}}\right)^{j}}\right) \frac{T_{1}}{S_{j}^{j}}=\sum_{j=1}^{n}\left(\sum_{k_{j}} \frac{T_{1}}{\left(x-\alpha_{k_{j}}\right)^{j}}\right)=F .
$$

Therefore Equation (7) is satisfied. Additionally, by Lemma $2 F_{j, j} \in K[x]$, and therefore $F_{j, j} \frac{T_{1}}{S_{j}^{j}}=F_{j, j} \prod_{\ell \neq j} S_{\ell}^{\ell} \in K[x]$. Thus $F \in K[x]$.

Computing $F$ requires (i) to compute the squarefree decomposition of $T_{1}$, (ii) for each squarefree factor $S_{j}$, compute the polynomial $F_{j, j}$ and (iii) use formula of Lemma 2 to obtain $F$. The computation of $N_{2}$ is summarized in Algorithm 1. The complexity estimates on the right correspond to upper bounds on the number of operations over $K$, where $n$ the number of squarefree factors of $T_{1}$. Details are written in the appendix. The estimates in Steps 7 or 9 dominate the overall cost. Correctness follows from the lemma hereafter:

Lemma 3. Let $\left(S_{1}, S_{2}, \cdots, S_{n}\right)$ be the squarefree decomposition of $T_{1}=\prod_{i=1}^{r}\left(x-\alpha_{i}\right)^{e_{i}} \in$ $K[x]$. If we apply Lemma 1 successively to $S_{1}, S_{2}^{2}, \cdots, S_{n}^{n}$, (instead of $T_{1}$ ) then we obtain $F_{1,1}, F_{2,2}, \cdots, F_{n, n}$ respectively. Regarding the polynomial $T_{2}$, we have:

$$
N_{2} \equiv \sum_{j=1}^{n}\left(F_{j, j} T_{2} \bmod \left\langle S_{j}^{j}\right\rangle\right) \prod_{\ell \neq j} S_{\ell}^{\ell} \bmod \left\langle T_{1}\right\rangle .
$$

Proof. This is clear from the definition of $N_{2} \equiv F T_{2} \bmod \left\langle T_{1}\right\rangle$ (Cf. Proposition 2, Corollary 1) and Lemma 2. 


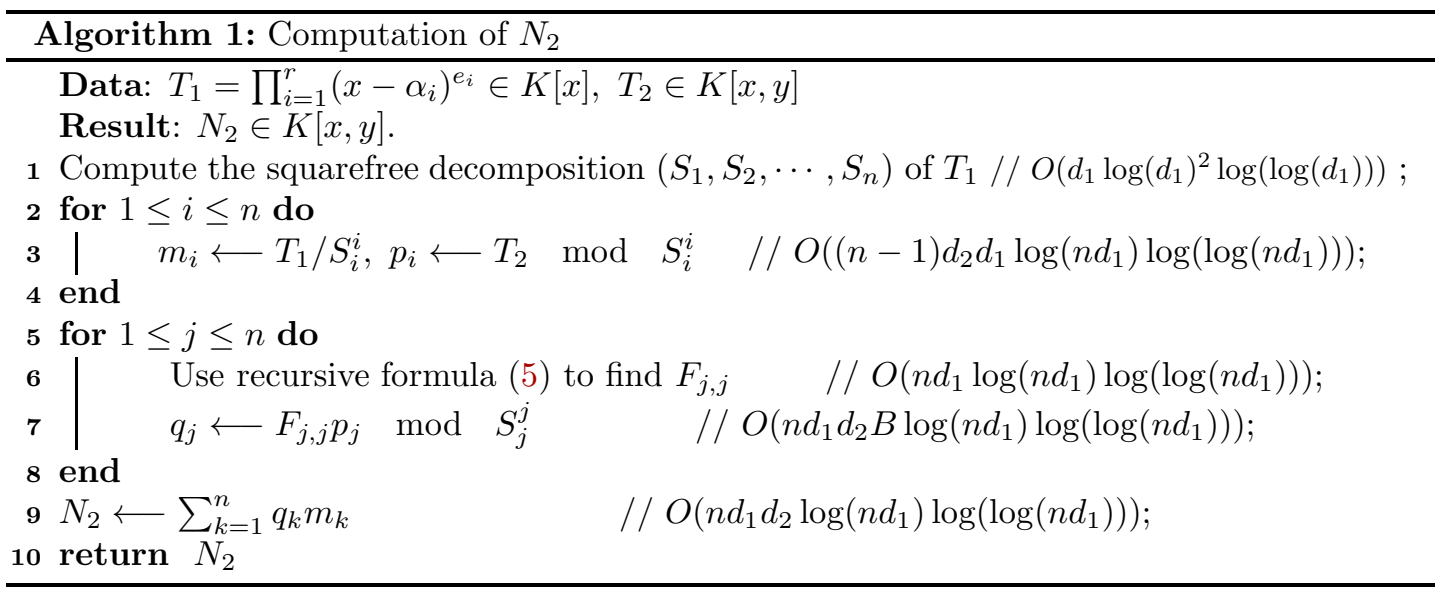

\section{Attempt of generalizations: case of three variables}

The treatment of the case of $n=2$ variables relies crucially on the fact that $T_{1} \in K[x]$ admits a (univariate) squarefree decomposition. This is more complicated for $T_{2} \in K[x, y]$ and we treat only special cases in this section.

Consider three variables $x, y, z$ ordered as $x \prec_{\text {lex }} y \prec_{\text {lex }} z$. We suppose that $T=\left\{T_{1}(x)\right.$, $\left.T_{2}(x, y), T_{3}(x, y, z)\right\}$ is lexicographic Gröbner basis of the 0 -dimensional ideal that it generates. Thus we have $\operatorname{LT}\left(T_{2}\right)=y^{d_{2}}, \operatorname{LT}\left(T_{3}\right)=z^{d_{3}}$ for integers $d_{2}>0, d_{3}>0$.

\subsection{Review on the case of a radical ideal in three variables}

Assumption 3. Let $T_{1}=\prod_{i=1}^{d_{1}}\left(x-\alpha_{i}\right)\left(\alpha_{i} \in \bar{K}\right.$, if $i \neq i^{\prime}$ then $\left.\alpha_{i} \neq \alpha_{i^{\prime}}\right)$ and $T_{2} \in K[x, y]$ be such that for $1 \leq i \leq d_{1} T_{2}\left(\alpha_{i}, y\right)=\prod_{j=1}^{d_{2}}\left(y-\beta_{i j}\right)\left(\beta_{i j} \in \bar{K}\right.$, if $j \neq j^{\prime}$ then $\left.\beta_{i j} \neq \beta_{i j^{\prime}}\right)$ is satisfied. Then the ideal generated by $T_{1}$ and $T_{2}$ is radical.

Theorem 2. 1) Under Assumption 3, we have:

$$
T_{3}(x, y, z)=\sum_{i=1}^{d_{1}}\left(\sum_{j=1}^{d_{2}} T_{3}\left(\alpha_{i}, \beta_{i j}, z\right) \prod_{j^{\prime} \neq j} \frac{y-\beta_{i j^{\prime}}}{\beta_{i j}-\beta_{i j^{\prime}}}\right) \prod_{i^{\prime} \neq i} \frac{x-\alpha_{i}^{\prime}}{\alpha_{i}-\alpha_{i}^{\prime}} .
$$

2) Define $F=\frac{d T_{1}}{d x}, G=\frac{\partial T_{2}}{\partial y}$. Then the following holds:

$$
F \cdot G \cdot T_{3} \equiv \sum_{i=1}^{d_{1}}\left(\sum_{j=1}^{d_{2}} T_{3}\left(\alpha_{i}, \beta_{i j}, z\right) \prod_{j^{\prime} \neq j}\left(y-\beta_{i j^{\prime}}\right)\right) \prod_{i^{\prime} \neq i}\left(x-\alpha_{i}^{\prime}\right) \bmod \left\langle T_{1}, T_{2}\right\rangle .
$$

3) Defining $N_{3}:=\mathrm{NF}_{\left\langle T_{1}, T_{2}\right\rangle}\left(F G T_{3}\right)$, the ideal equality holds: $\left\langle T_{1}, T_{2}, T_{3}\right\rangle=\left\langle T_{1}, N_{2}, N_{3}\right\rangle$.

Remark 5. Since $F \in K[x], G \in K[x, y]$ we also have $N_{3} \in K[x, y]$.

Moreover, as already noted in the case of two variables in Remarks 1, 3, by comparing $T_{3}$ and $N_{3}$ in (8) and (9) the terms $\prod_{j^{\prime} \neq j} \frac{1}{\beta_{i j}-\beta_{i j^{\prime}}} \prod_{i^{\prime} \neq i} \frac{1}{\alpha_{i}-\alpha_{i}^{\prime}}$ have been removed. 


\subsection{Toward generalization}

We do not assume that $T_{1}, T_{2}, T_{3}$ generate a radical ideal anymore. More precisely:

Assumption 4. Let us write $T_{1}=\prod_{i=1}^{r_{1}}\left(x-\alpha_{i}\right)^{e}\left(\alpha_{i} \in \bar{K}\right.$, if $i \neq i^{\prime}$ then $\left.\alpha_{i} \neq \alpha_{i^{\prime}}, e>0\right)$ in $\bar{K}[x]$. Concerning $T_{2}$, for $1 \leq i \leq r_{1}$, we assume that $T_{2} \bmod \left\langle\left(x-\alpha_{i}\right)^{e}\right\rangle$ is factorized over $\bar{K}[x, y]$, and that each factor comes with the same multiplicity: $T_{2} \equiv$

$\prod_{j=1}^{r_{2}}\left(y-g_{i, j}(x)\right)^{\ell} \bmod \left\langle\left(x-\alpha_{i}\right)^{e}\right\rangle,\left(g_{i, j} \in \bar{K}[x]\right.$, and $g_{i, j}(x) \not \equiv g_{i, j^{\prime}}(x) \bmod \left\langle\left(x-\alpha_{i}\right)^{e}\right\rangle$ if $\left.j \neq j^{\prime}\right)$.

Remark 6. Under Assumption 4, for any $1 \leq i \leq r_{1}$, define $M_{i}=T_{1} /\left(x-\alpha_{i}\right)^{e} \in \bar{K}[x]$. By Proposition 2, there exists $u_{i} \in \bar{K}[x]$ such that $\sum_{i=1}^{r_{1}} u_{i} M_{i}=1$ and

$$
T_{2} \equiv \sum_{i=1}^{r_{1}}\left(\prod_{j=1}^{r_{2}}\left(y-g_{i j}(x)\right)^{\ell}\right) u_{i} M_{i} \bmod \left\langle T_{1}\right\rangle \quad(\text { in } \bar{K}[x, y]) \text {. }
$$

We need another hypothesis, which was not necessary in the case of two variables (see Concluding Remarks "Discussion").

Assumption 5. On top of Assumption 4, suppose additionally

for all $1 \leq i \leq r_{1}, \quad$ when $j \neq j^{\prime}, \quad \operatorname{gcd}\left(\left(x-\alpha_{i}\right)^{e}, g_{i j}(x)-g_{i j^{\prime}}(x)\right)=1$ (in $\left.\bar{K}[x]\right)$.

Remark 7. This is equivalent to $\operatorname{gcd}\left(x-\alpha_{i}, g_{i j}(x)-g_{i j^{\prime}}(x)\right)=1$, or $g_{i j}\left(\alpha_{i}\right) \neq g_{i j^{\prime}}\left(\alpha_{i}\right)$.

Proposition 3. Under Assumptions 4,5, consider for $1 \leq i \leq r_{1}, 1 \leq j \leq r_{2}$ the notations $g_{i j}, M_{i}$ and $u_{i}$ of Remark 6. Define additionally $M_{i j}, u_{i j} \in \bar{K}[x, y]$ as follows:

$$
M_{i j} \equiv \frac{T_{2}}{\left(y-g_{i j}\right)^{\ell}} \bmod \left\langle\left(x-\alpha_{i}\right)^{e}\right\rangle, \text { and } \sum_{j} u_{i j} M_{i j} \equiv 1 \bmod \left\langle\left(x-\alpha_{i}\right)^{e}\right\rangle \text {, over } \bar{K}[x, y]
$$

Then, by denoting $p_{i j} \equiv T_{3} \bmod \left\langle\left(x-\alpha_{i}\right)^{e},\left(y-g_{i j}\right)^{\ell}\right\rangle \in \bar{K}[x, y, z], T_{3}$ satisfies:

$$
T_{3}(x, y, z) \equiv \sum_{i=1}^{r_{1}}\left(\sum_{j=1}^{r_{2}} p_{i j} u_{i j} M_{i j}\right) u_{i} M_{i} \bmod \left\langle T_{1}, T_{2}\right\rangle
$$

Equivalently $T_{3}=\mathrm{NF}_{\left\langle T_{1}, T_{2}\right\rangle}\left(\sum_{i=1}^{r_{1}}\left(\sum_{j=1}^{r_{2}} p_{i j} u_{i j} M_{i j}\right) u_{i} M_{i}\right)$.

Proof. Conditions related to $M_{i}$ and $u_{i}$ were already proved for the case of two variables in the previous section. Let us construct the polynomials $u_{i j} \in \bar{K}[x, y]$ satisfying Condition (10).

Denote $A=\bar{K}[x] /\left\langle\left(x-\alpha_{i}\right)^{e}\right\rangle$. By Assumption 5, for all $1 \leq i \leq r_{1}, 1 \leq j^{\prime}<j \leq r_{2}$, polynomials $g_{i j}(x)-g_{i j^{\prime}}(x)$ possess inverses in $A$,

$$
\begin{gathered}
y-g_{i j^{\prime}}=y-g_{i j}+g_{i j}-g_{i j^{\prime}} \quad \text { in } A[y] \\
\left(g_{i j}-g_{i j^{\prime}}\right)^{-1}\left(y-g_{i j^{\prime}}\right)-\left(g_{i j}-g_{i j^{\prime}}\right)^{-1}\left(y-g_{i j}\right)=1 \text { in } A[y]
\end{gathered}
$$

from which we get: $\left\langle y-g_{i j}(x)\right\rangle+\left\langle y-g_{i j^{\prime}}(x)\right\rangle=\langle 1\rangle$ in $A[y]$. Therefore polynomials $u_{i j}$ verifying Condition (10) exist.

Let us show that Equation (11) holds. For $1 \leq i \leq r_{1}$, according to the Chinese Remaindering Theorem (CRT) applied to Condition (10) gives: $T_{3} \equiv \sum_{j=1}^{r_{2}} p_{i j} u_{i j} M_{i j} \bmod \left\langle\left(x-\alpha_{i}\right)^{e}, T_{2}\right\rangle$. Similarly, the definition of $M_{i}, u_{i}$ yields again by the CRT: $T_{3} \equiv \sum_{i=1}^{r_{1}}\left(\sum_{j=1}^{r_{2}} p_{i j} u_{i j} M_{i j}\right) u_{i} M_{i}$ $\bmod \left\langle T_{1}, T_{2}\right\rangle$ which is Equation (11). 
Example 4. Defining $T_{1}=x^{2}(x-2)^{2}, T_{2}=\left(y-x^{2}\right)^{2}(y-1)^{2}$, we have:

$$
\left\{\begin{array}{l}
T_{2} \equiv y^{2}(y-1)^{2} \bmod \left\langle x^{2}\right\rangle \\
T_{2} \equiv(y-4 x+4)^{2}(y-1)^{2} \bmod \left\langle(x-2)^{2}\right\rangle
\end{array}\right.
$$

so that Assumption 5 is fulfilled. Define $\quad M_{2}=x^{2}$ and $\quad M_{1}=(x-2)^{2}$, as well as:

$$
\left\{\begin{array} { l } 
{ M _ { 1 2 } = y ^ { 2 } } \\
{ M _ { 1 1 } = ( y - 1 ) ^ { 2 } } \\
{ M _ { 2 2 } = ( y - 4 x + 4 ) ^ { 2 } } \\
{ M _ { 2 1 } = ( y - 1 ) ^ { 2 } }
\end{array} \quad \left\{\begin{array} { l } 
{ p _ { 1 1 } \equiv z \operatorname { m o d } \langle M _ { 2 } , M _ { 1 2 } \rangle } \\
{ p _ { 1 2 } \equiv z + 1 \operatorname { m o d } \langle M _ { 2 } , M _ { 1 1 } \rangle } \\
{ p _ { 2 1 } \equiv z + x \operatorname { m o d } \langle M _ { 1 } , M _ { 2 2 } \rangle } \\
{ p _ { 2 2 } \equiv z + y \operatorname { m o d } \langle M _ { 1 } , M _ { 2 1 } \rangle }
\end{array} \quad \left\{\begin{array}{l}
u_{11}=1+2 y \\
u_{12}=3-2 y \\
u_{21}=\frac{1}{27}(8 x y-18 y-32 x+75) \\
u_{22}=\frac{1}{27}(-8 x y+18 y+1)
\end{array}\right.\right.\right.
$$

Thus, for $1 \leq i \leq 2, \sum_{1 \leq j \leq 2} u_{i j} M_{i j} \equiv 1 \bmod \left\langle M_{i}\right\rangle$ hold. From these data, Proposition 3 gives:

$$
\begin{aligned}
T_{3} & \equiv \sum_{i=1}^{2} u_{i} M_{i}\left(\sum_{j=1}^{2} p_{i j} u_{i j} M_{i j}\right) \bmod \left\langle T_{1}, T_{2}\right\rangle \\
& \equiv u_{1} M_{2}\left(p_{11} u_{11} M_{12}+p_{12} u_{12} M_{11}\right)+u_{2} M_{1}\left(p_{21} u_{21} M_{22}+p_{22} u_{22} M_{21}\right) \bmod \left\langle T_{1}, T_{2}\right\rangle
\end{aligned}
$$

Therefore, after taking normal form modulo $T_{1}, T_{2}$ we get:

$$
T_{3}=z-\frac{19}{36} x^{3} y^{3}+\frac{169}{108} x^{2} y^{3}-2 y^{3}+x^{3} y^{2}-\frac{103}{36} x^{2} y^{2}+3 y^{2}-\frac{2}{3} x^{3} y+\frac{16}{9} x^{2} y+\frac{7}{36} x^{3}-\frac{13}{27} x^{2}
$$

We can check that the polynomial $T_{3}$ satisfies expressly $T_{3} \equiv p_{i j} \bmod \left\langle M_{i}, M_{i j}\right\rangle$ for $1 \leq i \leq 2,1 \leq j \leq 2$.

The following proposition follows from Proposition 3. The notations are the same. For $1 \leq i \leq r$ define $G_{i} \in \bar{K}[x, y]$ such that $G_{i} \equiv \sum_{j=1}^{r_{2}} M_{i j} \bmod \left\langle\left(x-\alpha_{i}\right)^{e}\right\rangle$ in $\bar{K}[x, y]$.

Proposition 4. By defining $F=\sum_{i=1}^{r_{1}} M_{i}, G=\sum_{i=1}^{r_{1}} G_{i} u_{i} M_{i}$ the following hold:

$$
F \in K[x], \quad G \in K[x, y], \quad F \cdot G \cdot T_{3} \equiv \sum_{i=1}^{r_{1}}\left(\sum_{j=1}^{r_{2}} p_{i j} M_{i j}\right) M_{i} \quad \bmod \left\langle T_{1}, T_{2}\right\rangle .
$$

Proof. The fact that $F \in K[x]$ and $G \in K[x, y]$ is proved in Lemma 6. From Proposition 3:

$$
T_{3} \equiv \sum_{j=1}^{r_{2}} p_{i j} u_{i j} M_{i j} \bmod \left\langle\left(x-\alpha_{i}\right)^{e}, T_{2}\right\rangle
$$

The fact that $M_{i j} M_{i j^{\prime}} \equiv 0 \bmod \left\langle\left(x-\alpha_{i}\right)^{\ell}, T_{2}\right\rangle$ if $j \neq j^{\prime}$ implies that:

$$
\begin{aligned}
G_{i} \cdot \sum_{j=1}^{r_{2}} p_{i j} u_{i j} M_{i j} & \equiv\left(\sum_{j=1}^{r_{2}} M_{i j}\right)\left(\sum_{j=1}^{r_{2}} p_{i j} u_{i j} M_{i j}\right) \bmod \left\langle\left(x-\alpha_{i}\right)^{e}, T_{2}\right\rangle \\
\equiv \sum_{j=1}^{r_{2}} p_{i j} u_{i j} M_{i j}^{2} & \equiv \sum_{j=1}^{r_{2}} p_{i j} M_{i j}\left(1-\sum_{t \neq j} u_{i t} M_{i t}\right) \bmod \left\langle\left(x-\alpha_{i}\right)^{e}, T_{2}\right\rangle \\
& \equiv \sum_{j=1}^{r_{2}} p_{i j} M_{i j} \bmod \left\langle\left(x-\alpha_{i}\right)^{e}, T_{2}\right\rangle .
\end{aligned}
$$

By the Chinese Remaindering Theorem,

$$
\sum_{i=1}^{r_{1}} u_{i} M_{i}\left(G_{i} \sum_{j=1}^{r_{2}} p_{i j} u_{i j} M_{i j}\right) \equiv \sum_{i=1}^{r_{1}} u_{i} M_{i}\left(\sum_{j=1}^{r_{2}} p_{i j} M_{i j}\right) \bmod \left\langle T_{1}, T_{2}\right\rangle .
$$

Therefore by denoting $G=\sum_{i=1}^{r_{1}} G_{i} u_{i} M_{i}$,

$$
G \cdot T_{3} \equiv \sum_{i=1}^{r_{1}} u_{i} M_{i}\left(\sum_{j=1}^{r_{2}} p_{i j} M_{i j}\right) \bmod \left\langle T_{1}, T_{2}\right\rangle
$$

holds. Thanks to Proposition $2 \quad F \cdot G \cdot T_{3} \equiv \sum_{i=1}^{r_{1}} M_{i}\left(\sum_{j=1}^{r_{2}} p_{i j} M_{i j}\right) \bmod \left\langle T_{1}, T_{2}\right\rangle$. 
The following Corollary is proved in a similar way as Corollary 1

Corollary 2. Defining $N_{3}:=\mathrm{NF}_{\left\langle T_{1}, T_{2}\right\rangle}\left(F \cdot G \cdot T_{3}\right)$, the following equality of ideals is satisfied:

$$
\left\langle T_{1}, T_{2}, T_{3}\right\rangle=\left\langle T_{1}, T_{2}, N_{3}\right\rangle=\left\langle T_{1}, N_{2}, N_{3}\right\rangle .
$$

Remark 8. 1. The fact that $N_{3} \in K[x, y, z]$ is proved in Lemma 6 .

2. From the definition of $N_{3}$, we see that $N_{3} \equiv \sum_{i=1}^{r_{1}}\left(\sum_{j=1}^{r_{2}} p_{i j} M_{i j}\right) M_{i} \bmod \left\langle T_{1}, T_{2}\right\rangle$. By comparing with $T_{3}$ thanks to Proposition 3 , the terms $u_{i j} \cdot u_{i}$ have been removed.

\subsection{Computation of $N_{3}$}

In this paragraph, is explained how to compute efficiently the polynomial $G$ of Proposition 4 under Assumption 3. The notations are the same as in Propositions 3,4.

Lemma 4. In Assumption \& when $\ell=1$ we have: $G \equiv \frac{\partial T_{2}}{\partial y} \bmod \left\langle T_{1}\right\rangle$.

Proof. From Remark 6 and the fact that $\ell=1$ :

$$
\frac{\partial T_{2}}{\partial y} \equiv \sum_{j=1}^{r_{2}} \prod_{k \neq j}\left(y-g_{i k}(x)\right) \equiv \sum_{j=1}^{r_{2}} M_{i j} \equiv G_{i} \quad \bmod \left\langle\left(x-\alpha_{i}\right)^{e}\right\rangle .
$$

By the Chinese Remaindering theorem, it follows that:

$$
\sum_{i=1}^{r_{1}} u_{i} M_{i}\left(\sum_{j=1}^{r_{2}} \prod_{k \neq j}\left(y-g_{i k}(x)\right)\right) \equiv \sum_{i=1}^{r_{1}} u_{i} M_{i} G_{i} \equiv G \bmod \left\langle T_{1}\right\rangle .
$$

Putting these equalities altogether, we obtain the equality stated.

Lemma 4 supplies with a straightforward method to compute $G$ and thus, according to the definition of $N_{3}$ in Corollary 2, to compute $N_{3}$ when $\ell=1$. We focus next on the case $\ell>1$ and the computation of $G$ is addressed in Lemma 7. Recall that from Proposition 4

$$
G=\sum_{i=1}^{r_{1}} u_{i} M_{i} G_{i} \equiv \sum_{i=1}^{r_{1}} u_{i} M_{i}\left(\sum_{j=1}^{r_{2}} \prod_{k \neq j}\left(y-g_{i k}(x)\right)^{\ell}\right) \bmod \left\langle T_{1}\right\rangle
$$

Since the term $\sum_{i=1}^{r_{1}} u_{i} M_{i}$ has been treated in the section dealing with the case of two variables, we only need to address the computation of $G_{i}$. The Lemma below is "local", i.e modulo $\left\langle\left(x-\alpha_{i}\right)^{e}\right\rangle$, and allows to compute $G_{i}$. The proof is similar to as the one of Lemma 1. Lemma 7 is a global version to compute $G$.

Lemma 5. Denote $S_{i}=\prod_{j=1}^{r_{2}}\left(y-g_{i j}(x)\right), G_{i, k}=\sum_{j=1}^{r_{2}} \prod_{j^{\prime} \neq j}\left(y-g_{i j^{\prime}}(x)\right)^{k}$. Note that $G_{i}=G_{i, \ell}$. Starting from $S_{i}, G_{i, \ell}$ can be computed by the following recurrence relation:

$$
G_{i, 1}=\frac{\partial S_{i}}{\partial y}, \quad G_{i, k+1}=G_{i, 1} \cdot G_{i, k}-\frac{S_{i}}{k} \cdot \frac{\partial G_{i, k}}{\partial y} \quad(1 \leq k \leq \ell-1) .
$$

Remark 9. Under Assumption 4, this Lemma is not of practical use, since we only need the "global" version of it stated in Lemma 7.

Lemma 6. The polynomial $N_{3}$ defined in Corollary 2 is in $K[x, y, z]$. 
Proof. By Lemma $2 F \in K[x]$ therefore we focus on proving that $G \in K[x, y]$. According to the definition of $N_{3}$, this is indeed sufficient to prove that $N_{3} \in K[x, y, z]$. Proposition 4 gives $G=\sum_{i=1}^{r_{1}} u_{i} M_{i}\left(\sum_{j=1}^{r_{2}} \prod_{k \neq j}\left(y-g_{i k}\right)^{\ell}\right) \bmod \left\langle T_{1}\right\rangle$. Consider the following isomorphism of algebras derived from the Chinese Remaindering Theorem:

$$
\begin{aligned}
\varphi: \oplus_{i=1}^{r_{1}}\left(\bar{K}[x] /\left\langle\left(x-\alpha_{i}\right)^{e}\right\rangle\right)[y] & \rightarrow\left(\bar{K}[x] /\left\langle T_{1}\right\rangle\right)[y] \\
\left(a_{1}, a_{2}, \cdots, a_{r_{1}}\right) & \mapsto \sum_{i=1}^{r_{1}} u_{i} M_{i} a_{i} .
\end{aligned}
$$

Here $S_{i}=\prod_{j=1}^{r_{2}}\left(y-g_{i, j}(x)\right) \in \bar{K}[x, y]$ and $T_{2} \equiv S_{i}^{\ell} \bmod \left\langle\left(x-\alpha_{i}\right)^{e}\right\rangle$. Let us define $S=$ $\varphi\left(S_{1}, S_{2}, \cdots, S_{r_{1}}\right)$. We have:

$$
\begin{aligned}
T_{2} & \equiv \sum_{i=1}^{r_{1}} u_{i} M_{i} S_{i}^{\ell} \equiv \varphi\left(S_{1}^{\ell}, S_{2}^{\ell}, \cdots, S_{r_{1}}^{\ell}\right) \bmod \left\langle T_{1}\right\rangle \\
& \equiv \varphi\left(S_{1}, S_{2}, \cdots, S_{r_{1}}\right)^{\ell} \equiv S^{\ell} \bmod \left\langle T_{1}\right\rangle
\end{aligned}
$$

By assumption, $T_{2} \in K[x, y]$ hence $S^{\ell} \in K[x, y]$ since that $T_{1} \in K[x]$. Combined with the fact that $S \in \bar{K}[x, y]$, it follows that $S \in K[x, y]$. Hence $\frac{\partial S}{\partial y} \in K[x, y]$, and by the next Lemma 7 $H_{1} \in K[x, y]$. In this way it is proved that $G\left(=H_{\ell}\right.$ in the next lemma $) \in K[x, y]$.

Lemma 7. For $t=1, \ldots, \ell$ let $H_{t}:=\varphi\left(G_{1, t}, G_{2, t}, \ldots, G_{r_{1}, t}\right)$, so that $H_{\ell}=G$. Let $S=$ $T_{2}^{1 / \ell} \bmod \left\langle T_{1}\right\rangle$ be as in the proof of Lemma 6 . These polynomials verify the recurrence relation:

$$
H_{1}=\frac{\partial S}{\partial y}, \quad \text { and } \quad H_{k+1}=H_{1} H_{k}-\frac{S}{k} \frac{\partial H_{k}}{\partial y} \quad(k \geq 1)
$$

Proof. Let $G_{i, j}=\sum_{j=1}^{r_{2}} \prod_{k \neq j}\left(y-g_{i k}\right)^{j}$ (as defined in Lemma 5). First let us handle $H_{1}$

$$
H_{1}=\sum_{i=1}^{r_{1}} u_{i} M_{i} G_{i, 1}=\sum_{i=1}^{r_{1}} u_{i} M_{i} \frac{\partial S_{i}}{\partial y}
$$

Since $u_{i} M_{i} \in \bar{K}[x]$, this polynomial does not depend on $y$ :

$$
H_{1}=\frac{\partial}{\partial y}\left(\sum_{i=1}^{r_{1}} u_{i} M_{i} S_{i}\right)=\frac{\partial \varphi\left(S_{1}, \cdots, S_{r_{1}}\right)}{\partial y}=\frac{\partial S}{\partial y} .
$$

Next let us treat the case $k \geq 1$ :

$$
\begin{aligned}
H_{k+1} & =\sum_{i=1}^{r_{1}} u_{i} M_{i} G_{i, k+1}=\sum_{i=1}^{r_{1}} u_{i} M_{i}\left(G_{i, 1} G_{i, k}-\frac{S_{i}}{k} \frac{\partial G_{i, k}}{\partial y}\right) \\
& =\varphi\left(G_{1,1} G_{1, k}-\frac{S_{1}}{k} \frac{\partial}{\partial y} G_{1, k}, \cdots, G_{r_{1}, 1} G_{r_{1}, k}-\frac{S_{r_{1}}}{k} \frac{\partial G_{r_{1}, k}}{\partial y}\right. \\
& =\varphi\left(G_{1,1}, \cdots, G_{r_{1}, 1}\right) \varphi\left(G_{1, k}, \cdots, G_{r_{1}, k}\right)-\frac{\varphi\left(S_{1}, \cdots, S_{r_{1}}\right)}{k} \frac{\partial}{\partial y} \varphi\left(G_{1, k}, \cdots, G_{r_{1}, k}\right)
\end{aligned}
$$

This latter equation is equal to $H_{1} H_{k}-\frac{S}{k} \frac{\partial H_{k}}{\partial y}$ which is what we wanted to show.

\section{Concluding remarks}

Summary of contributions We have extended the definitions of polynomials $N_{2}$, and to some extent $N_{3}$ of [4] to non-radical ideals that have a triangular set $\left\{T_{1}, T_{2}, T_{3}\right\} \subset K[x, y, z]$ as lexicographic Gröbner basis. A comparable decrease of the bit-size of coefficient has been 
observed in a Maple implementation. A major difference though lies in the use of squarefree decomposition, which, despite enjoying of fast algorithms in order to be computed, induces some complications that prevent to generalize these formulas in full generality to more than two variables. A detailed complexity analysis of the bit-size as done in [4] is planed in a later work, the present work focusing more on feasibility in two or three variables.

Implementation in Maple Algorithm 1 to compute the polynomial $N_{2}$ from $T_{1}$ and $T_{2}$ has been implemented in Maple. The squarefree decomposition algorithm is Yun's one taken from [6, Algorithm 14.21, p. 385] (see Section 2.4). The code occupies less than 40 lines. The table below shows benchmarks in the case where $T_{1}=(x-a)^{e_{1}}(x-b)^{e_{2}}$ for: (i) some random values $a, b \in \mathbb{Z}$ having 15 digits (ii) $e_{1}+e_{2}=22$, and $e_{1}$ is ranging from 1 to 11 (iii) moduli of $T_{2} \bmod \left\langle(x-a)^{e_{1}}\right\rangle$ and $T_{2} \bmod \left\langle(x-b)^{e_{2}}\right\rangle$ are chosen randomly of degree 15. It is indeed not necessary to consider more than two factors since this case was already treated in the previous work [4] which showed the decrease in the bit-size of coefficients. As we can see, the bit-size

\begin{tabular}{|l|l||l|l|l|l|l|l|l|l|l|l|l|}
\hline & $e_{1}$ & 1 & 2 & 3 & 4 & 5 & 6 & 7 & 8 & 9 & 10 & 11 \\
\hline \multirow{2}{*}{} & total & 232139 & 242837 & 234372 & 230948 & 222758 & 223658 & 225067 & 216909 & 219667 & 212003 & 191825 \\
& $\max$ & 575 & 612 & 583 & 550 & 544 & 544 & 543 & 512 & 512 & 494 & 471 \\
\hline \multirow{2}{*}{} & total & 61865 & 60738 & 60669 & 54793 & 58166 & 56831 & 54069 & 51230 & 49423 & 46876 & 44330 \\
& $\max$ & 328 & 311 & 300 & 260 & 269 & 255 & 239 & 225 & 211 & 197 & 179 \\
\hline
\end{tabular}

Table 1: Line total displays the sum of the number of digits over all the coefficients in $T_{2}$ (upper) or $N_{2}$ (lower). Line max displays the maximal number of digits ( $\approx$ bit-size) among all the coefficients in $T_{2}$ or $N_{2}$.

decrease observed get more important as both degrees $e_{1}$ and $e_{2}$ get higher: when $e_{1}=1$ and $e_{2}=21$, the ratio between the maximal bit-size of the coefficients of $N_{2}$ and the one of $T_{2}$ is $\frac{328}{575} \approx 0.57$ whereas the ratio becomes $\frac{179}{471} \approx 0.38$ for $e_{1}=e_{2}=11$. However better ratio than $1 / 3$ where not observed even for higher values of $e_{1}$ and $e_{2}$.

Discussion The algorithm to compute $N_{2}$ treated in Section 2 takes as input the polynomials $T_{1}, T_{2}$ and by means of efficient gcd-based subroutines output $N_{2}$. For $N_{3}$, Assumptions 5,4 are added. To be removed the following obstacles must be overcome:

Example 5. Let $T_{1}=x^{2}(x+1)^{2} \in \mathbb{Q}[x], T_{2}=\left(y-x^{2}\right)(y-x) \in \mathbb{Q}[x, y]$.

From $T_{2} \equiv y(y-x) \quad \bmod \left\langle x^{2}\right\rangle$, we see that $\operatorname{gcd}\left(x^{2}, 0-x\right)=\operatorname{gcd}\left(x^{2},-x\right) \neq 1$. If we set $A=K[x] /\left\langle x^{2}\right\rangle$, then:

$$
A[y] /\langle y(y-x)\rangle \not A[y] /\langle y\rangle \oplus A[y] /\langle y-x\rangle .
$$

On the other hand, since $T_{2} \equiv(y+2 x+1)(y-x) \quad \bmod \left\langle(x+1)^{2}\right\rangle$, we have $\operatorname{gcd}((x+$ $\left.1)^{2},-2 x-1-x\right)=\operatorname{gcd}\left((x+1)^{2},-3 x-1\right)=1$. If we write $B=K[x] /\left\langle(x+1)^{2}\right\rangle$, then:

$$
B[y] /\langle(y+2 x+1)(y-x)\rangle \simeq B[y] /\langle y+2 x+1\rangle \oplus B[y] /\langle y-x\rangle .
$$

In this example, Assumption 5 is verified in Equation (15) but not in Equation (14). Actually, the ring $A[y] /\langle y(y-x)\rangle$ is primary so we cannot decompose it furthermore. We have no algorithmic solution for this kind of input.

As for the restriction implied by the "same multiplicity" assumption 4, it can be loosened up to: denoting $T_{1}=\prod_{i} S_{i}^{e_{i}}$ the squarefree decomposition of $T_{1}$, then $T_{2} \equiv \prod_{j}\left(y-g_{i j}(x)\right)^{\ell_{i}} \bmod$ $\left\langle S_{i}^{e_{i}}\right\rangle$, with $g_{i j} \in \bar{K}[x]$ and verifying Assumption 5. Note that Assumption 4 states that $i=1$, and thus $\ell=\ell_{1}$. Example 5 above does not fulfill this generalized condition on $T_{2}$. 


\section{References}

[1] P. Aubry, D. Lazard, and M. Moreno Maza. On the theories of triangular sets. J. of Symbolic Computation, 28(1,2):45-124, 1999.

[2] C. Chen and M. Moreno Maza. Algorithms for computing triangular decompositions of polynomial systems. In Proceedings of the 36th International Symposium on Symbolic and Algebraic Computation, ISSAC '11, pages 83-90, New York, NY, USA, 2011. ACM.

[3] X. Dahan, M. Moreno Maza, É. Schost, W. Wu, and Y. Xie. Lifting techniques for triangular decompositions. In ISSAC'05, pages 108-115. ACM, 2005.

[4] X. Dahan and É. Schost. Sharp estimates for triangular sets. In ISSAC '04: Proceedings of the 2004 International Symposium on Symbolic and Algebraic Computation, pages 103-110. ACM Press, 2004.

[5] X. Li, C. Mou, and D. Wang. Decomposing polynomial sets into simple sets over finite fields: The zero-dimensional case. Computers $\mathcal{E}$ Mathematics with Applications, 60(11):2983 - 2997, 2010.

[6] J. von zur Gathen and J. Gerhard. Modern computer algebra. Cambridge University Press, New York, NY, USA, 2003. Second Eddition.

[7] D. Wang. Elimination Methods. Texts \& Monographs in Symbolic Computation. Springer Vienna, 2012.

[8] W. T. Wu. On zeros of algebraic equations - an application of Ritt principle. Kexue Tongbao, 5:1-5, 1986.

\section{Appendix}

We detail here the complexity estimates written in Algorithm 1. Let us recall some notations; $d_{1}:=\operatorname{deg}\left(T_{1}\right), d_{2}=\operatorname{deg}_{y}\left(T_{2}\right)$, and $d_{S_{j}}=\operatorname{deg}\left(S_{j}\right)$ where $T_{1}=\prod_{j=1}^{n} S_{j}^{j}$ is the squarefree decomposition of $T_{1}$. In particular, the degree equality $d_{1}=\sum_{j} j d_{S_{j}}$ holds.

Following [6, p. 242], we will use multiplication time function denoted $\mathrm{M}(d)$. This is an upper bound on the maximal number of operations in the base field $K$ to perform the multiplication of two polynomials of degree at most $d$. Shönhage Strassen's version of Fast Fourier Transform provides the estimate $\mathrm{M}(d)=O(d \log (d) \log (\log (d)))$. $\mathrm{M}($.$) is thus super-linear: \mathrm{M}(a)+\mathrm{M}(b) \leq$ $\mathrm{M}(a+b)$. We start by establishing the complexity stated in Remark 4.

Lemma 8. To compute $F_{e, e}$ from $S=\operatorname{sqf}\left(T_{1}\right)$ using the recurrence relation of Lemma 1 , less than $O(A \log (A) \log (\log (A)))$ operations over $K$ is required (where $A=(e+1)\left(d_{1}-e\right)$ ).

Proof. Let $\delta_{e, \ell}:=\operatorname{deg}\left(F_{e, \ell}\right)$. Starting from $\delta_{e, 1}=\operatorname{deg}\left(F_{e, 1}\right)=\operatorname{deg}(S)=r-1\left(\right.$ where $\left.r=d_{1} / e\right)$ the recurrence relation gives $\delta_{e, \ell+1}=\operatorname{deg}\left(F_{e, 1}\right)+\operatorname{deg}\left(F_{e, \ell}-1\right)=\delta_{e, 1}+\delta_{e, \ell}=r-1+\delta e, \ell$, yielding $\delta_{e, \ell}=(r-1) \ell=\ell d_{1} / e-\ell$.

The addition in the recurrence formula has indeed a negligible cost. For each $\ell=0, \ldots, e-$ 1 , we need to perform one differentiation of a polynomial of degree $\delta_{e, \ell}=\ell d_{1} / e-\ell$; one multiplication of a polynomial of degree $\delta_{e, \ell}-1$ by one of degree $r=d_{1} / e$; and one multiplication by two polynomials of respective degree $r-1=d_{1} / e-1$ and $\delta_{e, \ell}$. The differentiation is negligible, remains to evaluate the cost of the two multiplications: $\mathrm{M}\left(\delta_{e, \ell}-1\right)+\mathrm{M}\left(\delta_{e, \ell}\right) \leq 2 \mathrm{M}\left(\delta_{e, \ell}\right)$. This is repeated $e$ times yielding $\sum_{\ell=1}^{e} 2 \mathrm{M}\left(\delta_{e, \ell}\right) \leq 2 \mathrm{M}\left(\sum_{\ell=1}^{e} \ell\left(d_{1} / e-1\right)\right)=2 \mathrm{M}\left(\frac{e(e+1)}{2}\left(\frac{d_{1}}{e}-1\right)\right) \leq$ $\mathrm{M}\left((e+1)\left(d_{1}-e\right)\right)$ by super-linearity of $\mathrm{M}($.$) . The complexity of the lemma follows.$

Theorem 3. (a) Step 1 requires less than $O\left(d_{1} \log \left(d_{1}\right)^{2} \log \left(\log \left(d_{1}\right)\right)\right)$ operations in $K$.

(b) The cost of the for loop at Steps 2-4 is less than $O\left((n-1) d_{1} d_{2} \log \left(n d_{1}\right) \log \left(\log \left(n d_{1}\right)\right)\right)$.

(c) Step 6 requires up to $\mathrm{M}\left((j+1)\left(j d_{S_{j}}-j\right)\right)$ operations in $K$. 
(d) Step 7 can be performed within $d_{2}\left[\mathrm{M}\left(C_{j}\right)+4 \mathrm{M}\left(B_{j}\right)+\mathrm{M}\left(j d_{S_{j}}\right)+O\left(j d_{S_{j}}\right)\right]$ operations in $K$, where $C_{j}=\max \left\{d_{S_{j}}-j, d_{1}-j d_{S_{j}}\right\}$ and $B_{j}=d_{1}-j\left(d_{S_{j}}+1\right)$.

(e) The cost of the for loop of Steps 5-8 is less than $O\left(n d_{2} d_{1} \log \left(n d_{1}\right) \log \left(\log \left(n d_{1}\right)\right)\right)$.

(f) Step 9 requires at most $O\left(n d_{2} d_{1} \log \left(n d_{1}\right) \log \left(\log \left(n d_{1}\right)\right)\right)$ operations in $K$.

(g) the total cost, in term of number operations in $K$, of Algorithm 1 is roughly upper bounded by $O\left(n d_{2} d_{1} \log \left(n d_{1}\right) \log \left(\log \left(n d_{1}\right)\right)\right)$.

Proof. (a) is explained at the beginning of Section 2.4.

Fast Newton's iteration based Euclid algorithm [6, Theorem 9.6] allows to perform the division $T_{1} / S_{i}^{i}$ in $4 \mathrm{M}\left(\operatorname{deg}\left(T_{1}\right)-\operatorname{deg}\left(S_{i}^{i}\right)\right)+\mathrm{M}\left(\operatorname{deg}\left(S_{i}^{i}\right)\right)+O\left(\operatorname{deg}\left(S_{i}^{i}\right)\right)$. The loop runs over the number of squarefree factors $n$ of $T_{1}$, yielding the following estimate using the notations stated before the theorem, deduced from the super-linearity of $M($.$) ,$

$$
\sum_{i=1}^{n} 4 \mathrm{M}\left(d_{1}-i d_{S_{i}}\right)+\mathrm{M}\left(i d_{S_{i}}\right)+O\left(i d_{S_{i}}\right) \leq 4 \mathrm{M}\left((n-1) d_{1}\right)+\mathrm{M}\left(d_{1}\right)+O\left(d_{1}\right) .
$$

The dominating term of the above function can be bounded by $O\left((n-1) d_{1} \log \left(n d_{1}\right) \log \left(\log \left(n d_{1}\right)\right)\right)$.

The second operation performed during the loop of Steps 2-4 is $T_{2} \bmod S_{i}^{i}$, which amounts to execute $d_{2}$ Euclidean divisions, one for each coefficient of $T_{2} \in(K[x])[y]$. A similar analysis done for the first operation $T_{1} / S_{i}^{i}$ shows that this requires up to $O\left((n-1) d_{1} d_{2} \log \left(n d_{1}\right) \log \left(\log \left(n d_{1}\right)\right)\right)$ operations over $K$. This shows (b).

The estimate of Step 6 stated in (c) is explained in Lemma 8.

Note that $\operatorname{deg}\left(F_{j, j}\right)=\delta_{j, j}=j\left(d_{S_{j}} / j-1\right)=d_{S_{j}}-j$ and that each coefficient in $x$ of $p_{j} \in(K[x])[y]$ has degree at most $d_{1}-j d_{S_{j}}$. The multiplication $F_{j, j} p_{j}$ thus costs at most $d_{2} \mathrm{M}\left(C_{j}\right), C_{j}:=\max \left\{d_{S_{j}}-j, d_{1}-j d_{S_{j}}\right\}$. Since each coefficient in $x$ of $F_{j, j} p_{j}(x, y)$ is thereby of degree in $x$ less than $B_{j}:=d_{1}-j\left(d_{S_{j}}+1\right)$ it follows that the reduction $\bmod S_{j}^{j}$ of each of these coefficients can be estimated to cost less than $d_{2}\left[4 \mathrm{M}\left(d_{1}-j\left(d_{S_{j}}+1\right)\right)+\mathrm{M}\left(j d_{S_{j}}\right)+O\left(j d_{S_{j}}\right)\right]$. All in all, we can see that Step 7 can be performed within the number of operations over $K$ stated in (d).

To estimate the overall cost of the for loop at steps 5-8, it suffices to sum up the complexities of Steps 6 and 7 above. For Step 6: $\sum_{j=1}^{n} \mathrm{M}\left((j+1)\left(d_{S_{j}}-j\right)\right) \leq \mathrm{M}\left(\sum_{j=1}^{n}(j+1)\left(d_{S_{j}}-j\right)\right)$. We observe that $\sum_{j=1}^{n}(j+1)\left(d_{S_{j}}-j\right)=d_{1}+\left(\sum_{j} d_{S_{j}}\right)-(n(n+1) / 2)$, yielding $\mathrm{M}\left(d_{1}+\left(\sum_{j} d_{S_{j}}\right)-\right.$ $(n(n+1) / 2))$.

For Step 7: after summing up over $j$ the three terms $\mathrm{M}\left(C_{j}\right), 4 \mathrm{M}\left(B_{j}\right)$ and $\mathrm{M}\left(j d_{S_{j}}\right)$ involved in the estimate of (d), we obtain $\mathrm{M}\left((n-1) d_{1}+\tilde{C}_{k}\right), 4 \mathrm{M}\left((n-1) d_{1}-\left(\sum_{j} d_{S_{j}}\right)\right)$ and $\mathrm{M}\left(d_{1}\right)$ respectively (here $\tilde{C}_{k}=0$ if $C_{j}=d_{1}-j d_{S_{j}}$ for all $j$, or $\tilde{C}_{k}=d_{S_{k}}-k-\left(d_{1}-k d_{S_{k}}\right)$ if for some, necessarily unique $\left.k, C_{k}=d_{S_{k}}-k\right)$. As one can see, the dominating term is $\mathrm{M}\left(n\left(d_{1}-1\right)+\tilde{C}_{k}\right) \leq \mathrm{M}\left(n d_{1}\right)$. It is multiplied by $d_{2}$, largely outclassing the cost of Step 6 . Therefore, the overall cost of Step 6 and Step 7 is dominated by a term is $d_{2} \mathrm{M}\left(n d_{1}\right)$ yielding the upper bound of (e).

Finally Step 9 consists in multiplying the $d_{2}$ coefficients in $x$ of $q_{k} \in(K[x])[y]$ by $m_{k}$. The degrees at play are respectively $k d_{S_{k}}$ and $d_{1}-k d_{S_{k}}$, yielding a $d_{2} \mathrm{M}\left(C_{k}\right)$ where $C_{k}=$ $\max \left\{d_{1}-k d_{S_{k}}, k d_{S_{k}}\right\}$. Let $\mathcal{I}:=\left\{1 \leq k \leq n \mid k d_{S_{k}}=\max _{j}\left\{j d_{S_{j}}\right\}\right\}$. If $C_{k}=k d_{k}$ for some $k$, then $d_{1}-k d_{S_{k}}=\sum_{j \neq k} j d_{j} \leq k d_{k}$ showing that $k \in \mathcal{I}$ and $\mathcal{I}=\{k\}$.

It remains to sum over $j=1, \ldots, n$. As for the multiplication, in the case where $C_{k}=$ $d_{1}-k d_{S_{k}}$ for all $k$, we obtain the upper bound $d_{2} \mathrm{M}\left((n-1) d_{1}+\tilde{C}_{k}\right)$, where $\tilde{C}_{k}=0$ if for all $j, C_{j}=d_{1}-j d_{S_{j}}$, and $\tilde{C}_{k}=2 k d_{S_{k}}-d_{1}$ if there is a (unique) $k$ for which $C_{k}=k d_{k}$. This is upper bounded by $d_{2} \mathrm{M}\left(n d_{1}\right)$ and thus by $O\left(n d_{2} d_{1} \log \left(n d_{1}\right) \log \left(\log \left(n d_{1}\right)\right)\right)$ as stated.

We can see that Step 7 or Step 9 in worst case outclasses other steps, yielding the result (g). 